\title{
Enhancing 5-Fluorouracil Efficacy in a Primary Colorectal Cancer by Long-lasting Calcium Supplementation
}

\author{
KEUN-YEONG JEONG ${ }^{1 *}$, MINHEE PARK $^{1 *}$, IN UN KIM ${ }^{2 *}$, JAE JUN SIM $^{2}$ and HWAN MOOK KIM ${ }^{2}$ \\ ${ }^{1}$ Oncometplus Pharmaceuticals Co. R\&D division, Incheon, Republic of Korea; \\ ${ }^{2}$ Gachon Institute of Pharmaceutical Science, Gachon University, Incheon, Republic of Korea
}

\begin{abstract}
Background/Aim: 5-Fluorouracil (5-FU) over-use has led to an urgent need for alternative treatment regimens, such as a lower concentration of the drug because of its toxic effects. The aim of this study was to investigate the possibility of improving the antitumor effect of 5-FU without toxicity by targeting primary colorectal cancer $(C R C)$ with sustained calcium supplementation. Materials and Methods: The viability of CRC cells was determined after treatment of 5$F U$, lactate calcium salt (CaLac), or the combination of te two. Western blot analysis for the focal adhesion kinase (FAK) signaling cascade was performed to investigate the underlying mechanism. A xenograft model was established to evaluate antitumor efficacy of each treatment, and the necrotic effect was also observed in tumor tissues. Results: By the combined treatment, proteolysis of FAK signaling cascade, was mediated by sustained calcium supplementation resulting in further decrease in the clonogenicity of CRC cells. The in vivo anticancer efficacy including tumor necrosis was significantly increased by the combination treatment compared to single treatment of with 5-FU. Conclusion: Sustained calcium supplementation was able to enhance the potency of 5-FU targeting the primary CRC.
\end{abstract}

Colorectal cancer (CRC) is the third most commonly diagnosed cancer and the third leading cause of cancerrelated death worldwide (1). Nearly 1.4 million people were

*These authors contributed equally to this work.

Correspondence to: Prof. Hwan Mook Kim, Gachon Institute of Pharmaceutical Sciences, Gachon University, Incheon, 21936, Republic of Korea. Tel: +82 328996446, Fax: +82 328204829, e-mail: hwanmook@gachon.ac.kr and Prof. Keun-Yeong Jeong, Oncometplus Pharmaceuticals Co., R\&D Division, Yeonsu-Gu, Incheon, 22006, Republic of Korea. Tel: +82 322050541, Fax: +82 322050542, e-mail: alvirus@naver.com

Key Words: Primary colorectal cancer, 5-FU, lactate salt, focal adhesion kinase, epithelial-mesenchymal transition, combination treatment. diagnosed with CRC in 2012, with the Republic of Korea displaying the highest incidence rates, followed by Slovakia and Hungary (2).

5-Fluorouracil (5-FU) is usually administered in patients diagnosed with CRC (3). Combination treatments with oxaliplatin and irinotecan are also conducted as a second-line regimen (4). However, the toxic effects of the treatment (5) have led to urgent need for alternative treatment regimens, such as a lower concentration of drugs.

Many considerations related to CRC treatment have been investigated. Recent studies indicated that dietary calcium supplementation plays a key role in the prevention of colon carcinogenesis (6) and also reduces the risk of CRC (7). However, controversy still exists regarding the effectiveness of calcium supplementation in CRC prevention. Thus, further research work is required to confirm the effect of calcium supplementation on CRC.

In medicine, Lactate calcium salt (CaLac) is most commonly used as a calcium supplement for the treatment of calcium deficiencies (8). The neutral chemical structure confers the remarkable property of being able to easily diffuse into cells without a specific ion channel. It has been reported that CaLac treatment leads to a significant calcium intake to CRC cells (9).

In this study, we used human-derived CRC cells to investigate the possibility of enhancing the antitumor efficacy of 5-FU by sustained calcium supplementation, even for a dose of 5-FU lower than that used in clinical practice.

\section{Materials and Methods}

Cell culture and reagents. Human CRC cell lines (HT-29 and HCT116) were purchased from the American Type Culture Collection (Manassas, VA, USA). Cells were maintained in RPMI-1640 medium (Welgene, Daegu, South Korea) in a humidified atmosphere of 5\% $\mathrm{CO}_{2}$, at $37^{\circ} \mathrm{C}$. 5-FU, CaLac were purchased from Sigma-Aldrich ( $\mathrm{St}$ Louis, MO, USA). Primary and secondary anti-rabbit antibodies for western blot analysis were purchased from Cell Signaling Technology (Danvers, MA, USA). For the western blot analysis, the primary antibodies recognized the following proteins: FAK, Src, Akt, and Glyceraldehyde 3-phosphate dehydrogenase (GAPDH). 
Colony formation assay. CRC cells were seeded at a density of $2.5 \times 10^{2}$ cells/well in a normal 6-well plate. After $24 \mathrm{~h}$, cells were treated with $2.5 \mathrm{mM}$ CaLac, $1 \mu \mathrm{M} 5-\mathrm{FU}$, or a combination of the two. After 14 days, the colonies were fixed with methanol and stained using hematoxylin (Thermo Fisher Scientific, Waltham, MA, USA). Grown colonies were counted under an optical microscope (Olympus, Center Valley, PA, USA).

Western blot analysis. CRC cells were washed twice with cold PBS and lysed with lysis buffer (20 mM Tris- $\mathrm{HCl}(\mathrm{pH} 7.6), 1 \mathrm{mM}$ EDTA, $140 \mathrm{mM} \mathrm{NaCl}, 1 \%$ Nonidet P-40, $1 \%$ aprotinin, $1 \mathrm{mM}$ phenylmethylsulfonyl fluoride, and $1 \mathrm{mM}$ sodium vanadate). $20 \mu \mathrm{g}$ protein extracts were loaded on $10 \%$ polyacrylamide gels. Separated proteins were transferred onto polyvinylidene fluoride membranes (Merck Millipore, Billerica, MA, USA). After blocking in 5\% nonfat milk (Bio-Rad, Hercules, CA, USA) for $1 \mathrm{~h}$, membranes were incubated overnight at $4^{\circ} \mathrm{C}$ with primary antibody diluted in TBST containing $100 \mathrm{mM}$ Tris- $\mathrm{HCl}(\mathrm{pH} 7.5), 1.5 \mathrm{M} \mathrm{NaCl}$, and $0.5 \%$ Tween-20 (Sigma-Aldrich, St Louis, MO, USA), to which 5\% BSA and $0.1 \%$ sodium azide (Sigma-Aldrich, St Louis, MO, USA) had been added. The specific dilutions for each antibody were as follows: FAK: 1:1000; Src: 1:1000; Akt: 1:1000; and GAPDH: 1:5000. On the next day, membranes were washed with TBST and incubated for $2 \mathrm{~h}$ with anti-rabbit secondary antibody (1:5000). Immunoblots were exposed to x-ray film (Agfa, Leverkusen, Germany) according to the manufacturer's protocol.

Xenograft animal model. All experiments were performed under the institutional guidelines established by the Institutional Animal Care and Use Committee at Gachon university (IACUC-2014-0055). 6week old $\mathrm{BALB} / \mathrm{c}$ nude mice were purchased from Charles River Breeding Laboratories (Wilmington, MA, USA). All animals were maintained in a 12 -hour light/dark cycle (light on, 08:00) at $22-25^{\circ} \mathrm{C}$ with free access to food and water. HCT-116 cells were injected $\left(1 \times 10^{7}\right.$ cells) into the dorsal flank to investigate the antitumor efficacy of 5-FU (50 mg/kg, intravenous; I.V.), CaLac ( $2 \mathrm{mg} / \mathrm{kg}$, subcutaneous; S.C.), or a combination of the two. Tumor sizes were measured three times a week, and volumes were calculated. At the end of experiment, tumors were isolated for the measurement of the weight or $\mathrm{H} \& \mathrm{E}$ staining.

Statistical analysis. All data are presented as the mean \pm standard deviation (SD). Statistical significance was analyzed using a Student's $t$-test depending on the normality of the data. A $p<0.05$ was considered to be statistically significant (Sigmastat ver. 3.5, Systat Software Inc., Chicago, IL, USA).

\section{Results}

Sustained calcium supplementation enhances the anticlonogenicity of 5-FU. Representative pictures for HT-29 and HCT-116 colonies are shown in Figure 1A and C. The combination treatment of CaLac and 5-FU resulted in a smaller colony size than the single treatments. The combination treatment also led to a reduction in the number of HT-29 colonies, from $67.67 \pm 7.23$ (control) to $52.33 \pm 5.13,35.33 \pm 6.35$, and 12.33 \pm 2.51 after treatment with $5-\mathrm{FU}, \mathrm{CaLac}$, and the combination treatment and to a reduction in the number of HCT-116 colonies, from $116.67 \pm 14.46$ (control) to $55 \pm 2$,
$21.33 \pm 4.04$, and 9.67 \pm 2.08 after treatment with 5-FU, CaLac, and the combination treatment, respectively (Figure 1B and D).

Sustained calcium supplementation mediates the inhibition of FAK signaling cascade. To determine the mechanism underlying the antitumor effect of sustained calcium supplementation, FAK signaling cascade was examined. Compared to control, single 5-FU treatment resulted in a slight increase in the levels of FAK (Figure 2A) The levels of Src and Akt was decreased without change in the levels of FAK following single treatment of HT-29 and HCT-116 cells with 5-FU (Figures $2 \mathrm{~B}$ and $\mathrm{C}$ ). In contrast, CaLac treatment clearly reduced the levels of FAK signaling molecules (Figure $2)$. Moreover, the results indicated that the combination treatment caused a clear decrease in the levels of FAK signaling molecules including Src and Akt, compared to the single treatment of 5-FU or CaLac (Figure 2).

Sustained calcium supplementation enhances antitumor effect of 5-FU on CRC. To investigate the in vivo efficacy of combination treatment, 5-FU was intravenously administered once a week for 3 weeks, and CaLac was subcutaneously administered 2 times daily for 3 weeks. Representative images of the tumor mass are shown in Figure 3A. In the group treated with 5-FU, tumor growth was significantly reduced to 2332.93 $\mathrm{mm}^{3}$ on the last day of measurement as compared to the control $\left(2965.65 \mathrm{~mm}^{3}\right)$, however the growth tended to recur after the end of the administration (Figure 3B). In the group treated with CaLac, tumor growth was significantly decreased to 1485.35 $\mathrm{mm}^{3}$ as compared to the control, and the growth rate of the tumor was kept slow after the end of the administration (Figure 3B). The tumor growth was significantly decreased to 802.01 $\mathrm{mm}^{3}$ by the combination treatment as compared to the control or the single treatments (Figure 3B). The tumor weight decreased to $1.50 \pm 0.25 \mathrm{~g}$ and $1.12 \pm 0.36 \mathrm{~g}$ after the single treatment of with 5-FU or CaLac, respectively, as compared to the control $(1.90 \pm 0.33 \mathrm{~g})$, and the combination treatment significantly decreased the tumor weight to $0.99 \pm 0.29 \mathrm{~g}$ (Figure $3 \mathrm{C})$. Histological analysis of the control group revealed high cell proliferation and a low rate of apoptosis, and partial and internal necrosis were observed after the individual treatment with 5-FU or CaLac. Following combination treatment, necrosis was observed in the whole area of the tumor mass, including the superficial part (Figure 4).

\section{Discussion}

In this study, calcium was consistently supplied using CaLac to investigate whether in enhances the antitumor efficacy of 5FU in the CRC following calcium-dependent reduction in FAK signaling molecules. The results demonstrated that sustained calcium supplementation enhanced the antitumor efficacy of 5$\mathrm{FU}$ by inducing necrosis in the whole tumor tissue. 
A
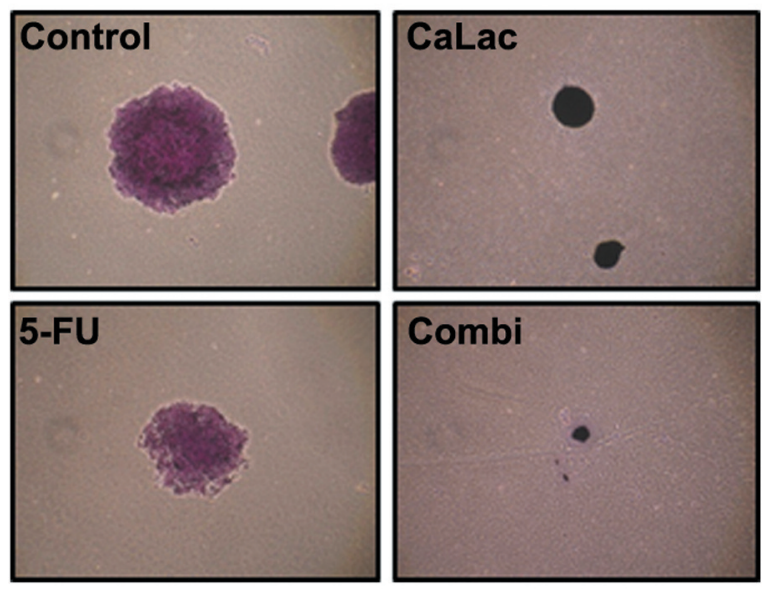

C
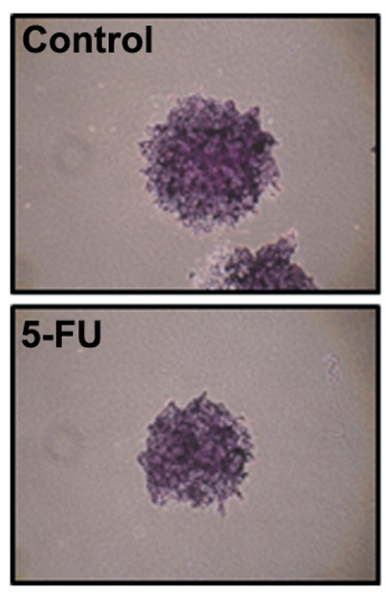
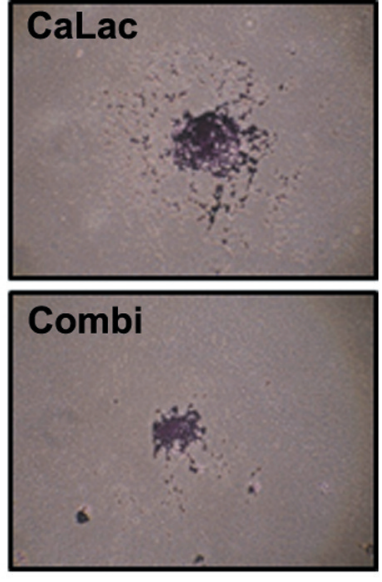

B

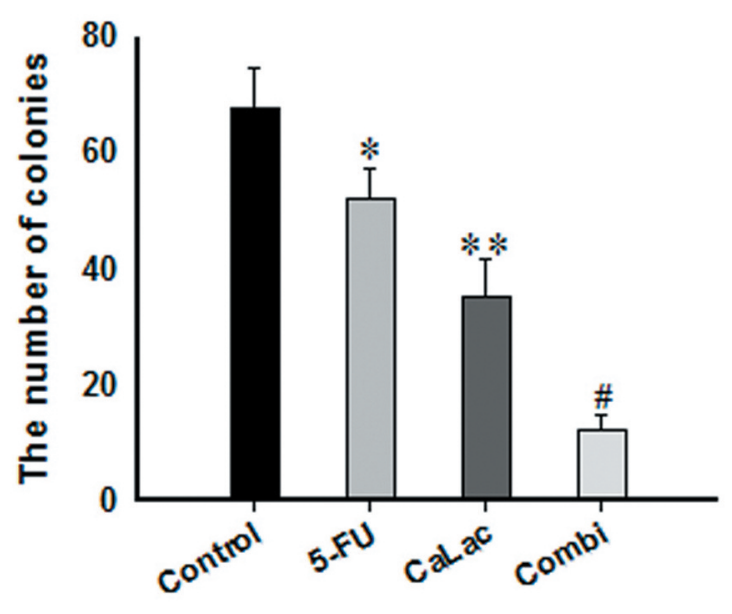

D

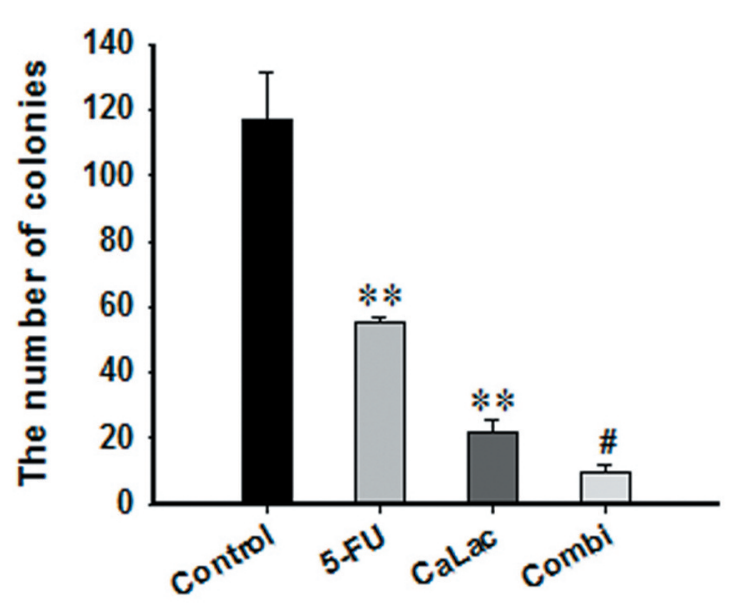

Figure 1. Clonogenic assay for in vitro antitumor effect. (A) The representative images of colonies. (B) Quantitative analysis for the number of colonies. ${ }^{*} p<0.05$ and ${ }^{* *} p<0.001 \mathrm{vs.} \mathrm{control,}{ }^{\#} p<0.05$ vs. the control, lactate calcium salt (CaLac), and 5-fluoruracil (5-FU) groups. Results are the mean $\pm S D$. Combi: Combination treatment.

5-FU inhibits access of deoxyuridine monophosphate to the nucleotide-binding site (10). The result induced deoxynucleotide pool imbalances and an increased level of deoxyuridine triphosphate, both of which are factors leading to DNA damage (3). According to our previous research, calcium influx causes calpain activation and proteolysis of FAK (11). FAK is involved in Src-mediated phosphorylation, which is necessary for the activation of Akt signaling. The signaling cascade of FAK, phosphatidylinositol 3-kinase (PI3K), and Akt plays an important role in the survival of cancer cells (12). Therefore, as the calcium effect on cancer cell is different from that of 5-FU (3), calcium supplementation would be an effective regimen to increase the efficacy of 5-FU, without a conflicting mechanism.
The rationale for the in vitro treatment of 2.5-mM CaLac and $1-\mu \mathrm{M} 5-\mathrm{FU}$ is based on the results on the inhibition of cell growth by different concentration of CaLac or 5-FU in our recent study (11). CaLac was at the optimal concentration for displaying the best antitumor effect. 5-FU was used at a very low concentration that has significant antitumor activity (11). We have chosen low concentrations to minimize the side effects of 5-FU (13).

Furthermore, the weekly usage dose in vivo is $50 \mathrm{mg} / \mathrm{kg}$ in mice via I.V. and the human equivalent dose is approximately $240 \mathrm{mg} / \mathrm{person} /$ day. This was a very low dose as compared to a regimen of up to $4,000 \mathrm{mg} /$ person/day that is used weekly to advanced gastric patients in a clinical setting. Our results 
A

HT-29

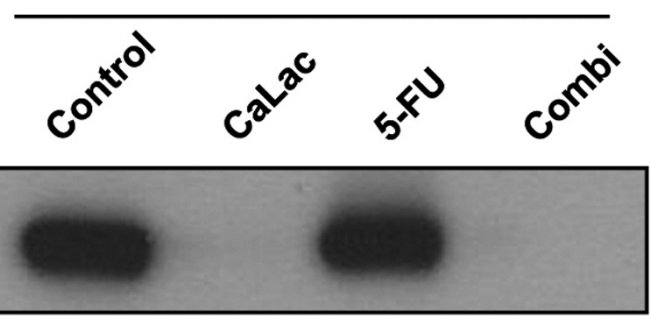

FAK

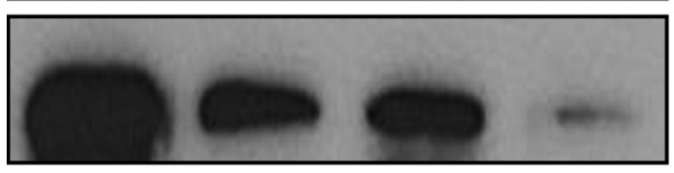

Src

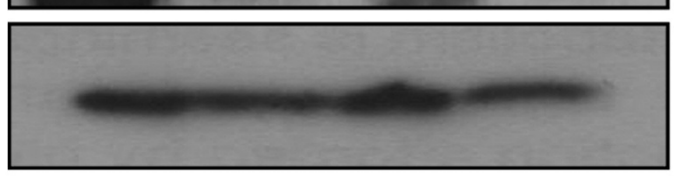

\section{HCT-116}
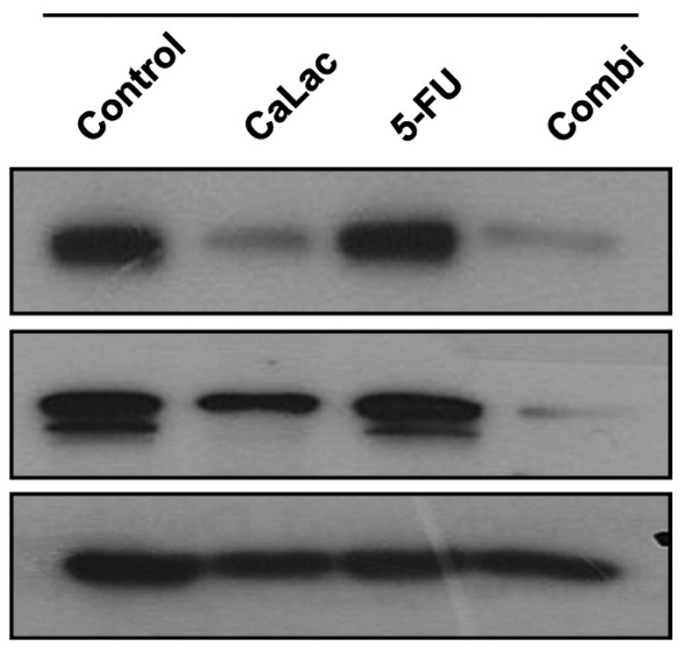

B

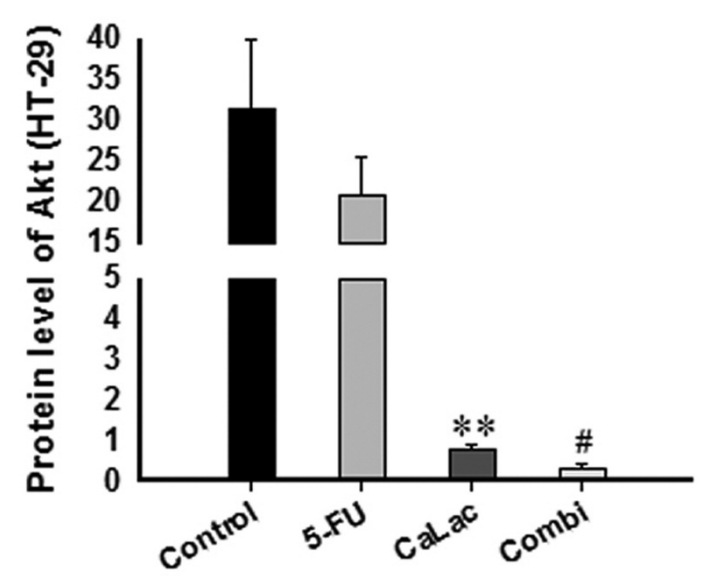

C

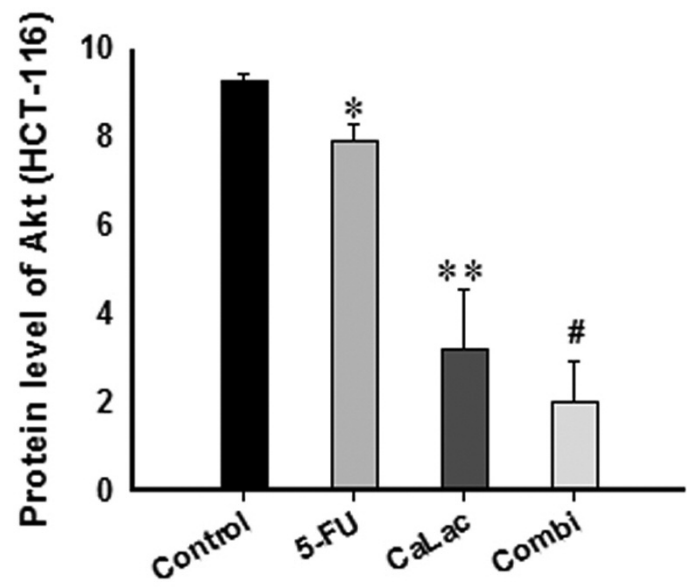

Figure 2. Western blot analysis for FAK signaling cascade elements. (A) Western blot analysis for FAK and Src levels. (B) Quantitative analysis for the Akt levels in HT-29. (C) Quantitative analysis for the Akt levels in HCT-116. *p<0.05 and **p<0.001 vs. control and 5-FU groups, ${ }^{*} p<0.05$ vs. control, CaLac, and 5-FU groups. Results are the mean \pm SD. Combi: Combination treatment.

indicated that sustained calcium supplementation enhanced the antitumor effect of 5-FU on primary CRC, which also suggested that the combined treatment may represent a means of reducing the required therapeutic doses of 5-FU (14).

In this study, calcium was continuously supplemented during the experiments. Despite the existence of numerous reports on the subject, controversy still exists regarding the effectiveness of calcium supplementation in CRC prevention $(15,16)$. Thus, further research including cells derived from a variety of patients with $\mathrm{CRC}$ is required to overcome the controversy on calcium supplementation and its effectiveness in CRC treatment.

\section{Conclusion}

The combination of 5-FU with calcium supplementation significantly inhibited CRC progression, even with a 5-FU dose below the clinical level. However, the use of calcium as a clinical therapy would require additional work to examine other parameters, such as application dose and route.

\section{Conflicts of Interest}

No potential conflicts of interest were disclosed. 
A
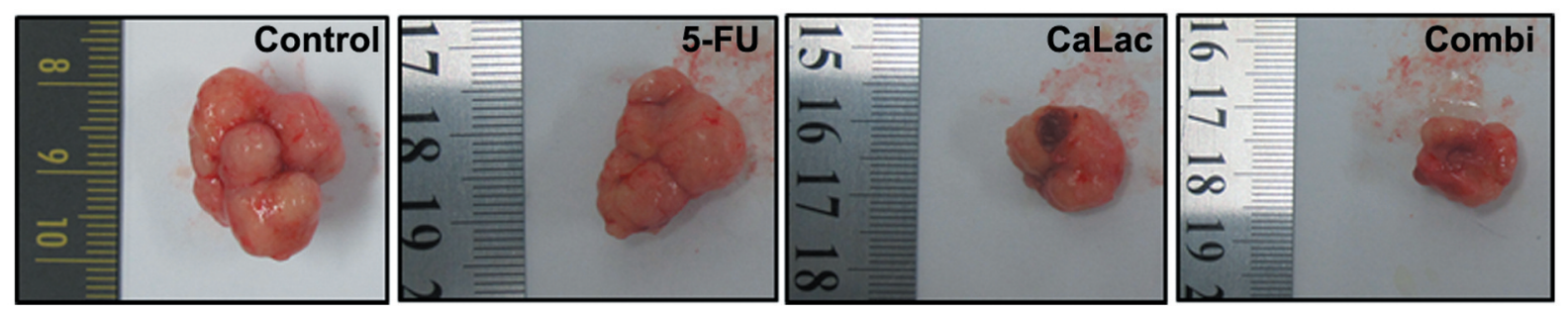

B

C
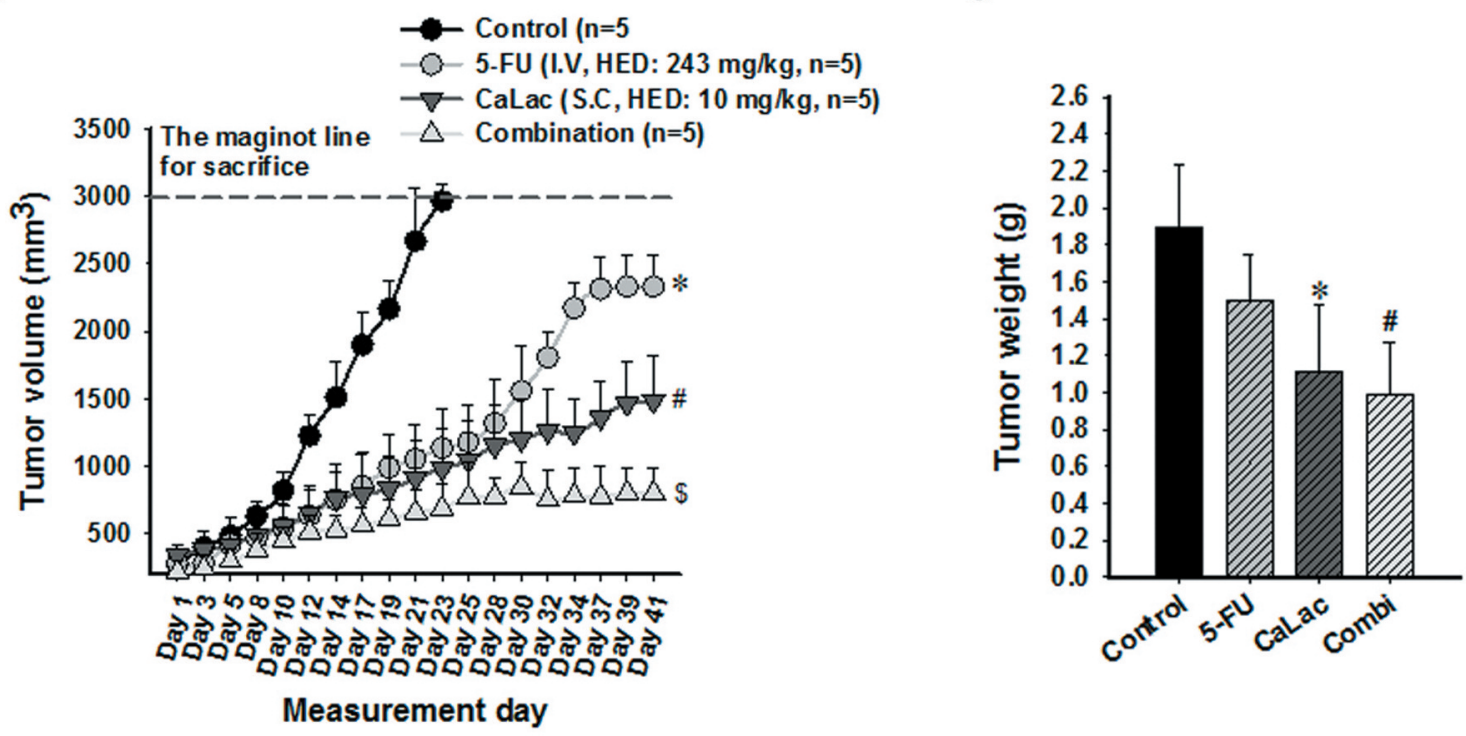

Figure 3. In vivo antitumor effect on CRC xenograft model. (A) Representative images of the tumor mass following autopsy. (B) Comparison of the tumor volume. (C) Quantitative analysis of the tumor weight. ${ }^{*} p<0.05 \mathrm{vs}$. control, ${ }^{\#} p<0.05$ vs. the control, and 5-FU groups. ${ }^{\$} p<0.001$ vs. the control, CaLac, and 5-FU groups. Results are the mean $\pm S D$. Combi: Combination treatment.

\section{Control}

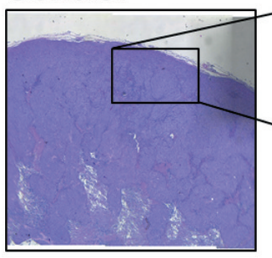

5-FU

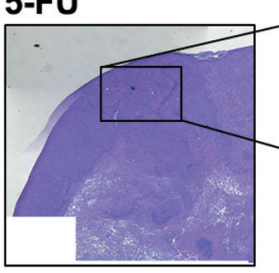

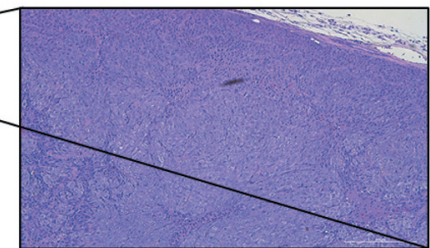

White line: partial necrosis

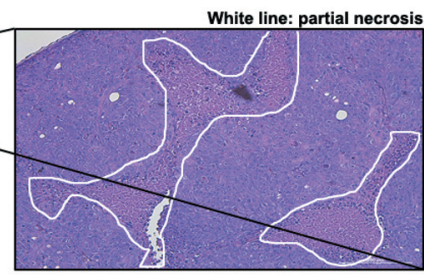

\section{CaLac}
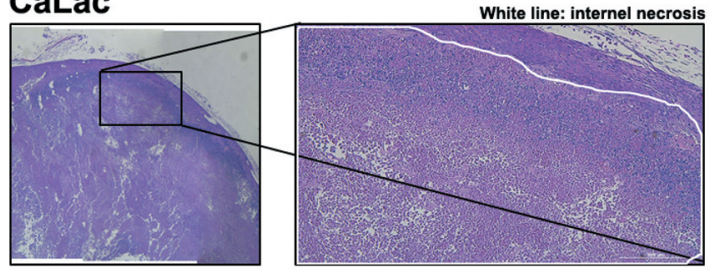

\section{Combi}
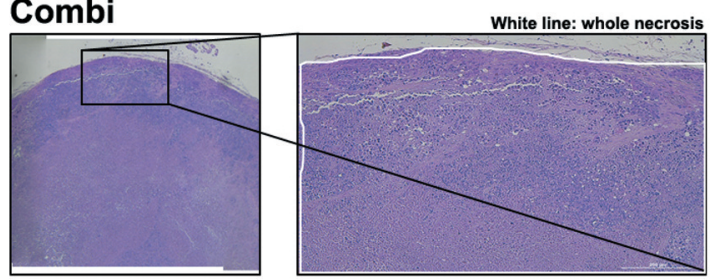

Figure 4. Histological analysis of tumor tissue. H\&E staining was performed to observe tumor cell differentiation and apoptosis and to select necrotic areas. 5-FU: Partial necrosis; CaLac: internal necrosis; Combination (Combi): whole necrosis, including the superficial part. 


\section{References}

1 Wheat CL, Clark-Snustad K, Devine B, Grembowski D, Thornton TA and Ko CW: Worldwide Incidence of Colorectal Cancer, Leukemia, and Lymphoma in Inflammatory Bowel Disease: An Updated Systematic Review and Meta-Analysis. Gastroenterol Res Pract 2016: 1632439, 2016.

2 Torre LA, Bray F, Siegel RL, Ferlay J, Lortet-Tieulent J and Jemal A: Global cancer statistics, 2012. CA Cancer J Clin 65: 87-108, 2015.

3 Longley DB, Harkin DP and Johnston PG: 5-fluorouracil: mechanisms of action and clinical strategies. Nat Rev Cancer 3: 330-338, 2003.

4 Calvo E, Cortes J, Rodriguez J, Fernandez-Hidalgo O, Rebollo $\mathrm{J}$ and Martin-Algarra S: Irinotecan, oxaliplatin, and 5fluorouracil/leucovorin combination chemotherapy in advanced colorectal carcinoma: a phase II study. Clin Colorectal Cancer 2: 104-110, 2002.

5 Lin YC, Chen JS, Wang CH, Wang HM, Chang HK, Liau CT, Yang TS, Liaw CC and Liu HE: Weekly high-dose 5-fluorouracil (5-FU), leucovorin (LV) and bimonthly cisplatin in patients with advanced gastric cancer. Jpn J Clin Oncol 31: 605-609, 2001.

6 Lamprecht SA and Lipkin M: Chemoprevention of colon cancer by calcium, vitamin $\mathrm{D}$ and folate: molecular mechanisms. Nat Rev Cancer 3: 601-614, 2003.

7 Park Y, Leitzmann MF, Subar AF, Hollenbeck A and Schatzkin A: Dairy food, calcium, and risk of cancer in the NIH-AARP Diet and Health Study. Arch Intern Med 169: 391-401, 2009.

8 Schafer AL and Shoback DM: Hypocalcemia: Diagnosis and Treatment. In: De Groot LJ, Beck-Peccoz P, Chrousos G, Dungan K, Grossman A, Hershman JM, Koch C, Korbonits M, McLachlan R, New M, Purnell J, Rebar R, Singer F and Vinik A (eds). South Dartmouth (MA) Endotext, 2000.

9 Sundaramoorthy P, Sim JJ, Jang YS, Mishra SK, Jeong KY, Mander P, Chul OB, Shim WS, Oh SH, Nam KY and Kim HM: Modulation of intracellular calcium levels by calcium lactate affects colon cancer cell motility through calcium-dependent calpain. PLoS One 10: e0116984, 2015.

10 Heidelberger C, Chaudhuri NK, Danneberg P, Mooren D, Griesbach L, Duschinsky R, Schnitzer RJ, Pleven E and Scheiner J: Fluorinated pyrimidines, a new class of tumourinhibitory compounds. Nature 179: 663-666, 1957.
11 Park M, Sundaramoorthy P, Sim JJ, Jeong KY and Kim HM: Synergistically anti-metastatic effect of 5-flourouracil on colorectal cancer cells via calcium-mediated focal adhesion kinase proteolysis. Anticancer Res 37: 103-114, 2017.

12 Thamilselvan V, Craig DH andBasson MD: FAK association with multiple signal proteins mediates pressure-induced colon cancer cell adhesion via a Src-dependent PI3K/Akt pathway. FASEB J 21: 1730-1741, 2007.

13 Focaccetti C, Bruno A, Magnani E, Bartolini D, Principi E, Dallaglio K, Bucci EO, Finzi G, Sessa F, Noonan DM and Albini A: Effects of 5-fluorouracil on morphology, cell cycle, proliferation, apoptosis, autophagy and ROS production in endothelial cells and cardiomyocytes. PLoS One 10: e0115686, 2015.

14 de Forni M, Malet-Martino MC, Jaillais P, Shubinski RE, Bachaud JM, Lemaire L, Canal P, Chevreau C, Carrié D, Soulié P, Roché H, Boudjema B, Mihura J, Martino R, Bernadet P and Bugat R: Cardiotoxicity of high-dose continuous infusion fluorouracil: a prospective clinical study. J Clin Oncol 10: 1795-1801, 1992.

15 Wactawski-Wende J, Kotchen JM, Anderson GL, Assaf AR, Brunner RL, O’Sullivan MJ, Margolis KL, Ockene JK, Phillips L, Pottern L, Prentice RL, Robbins J, Rohan TE, Sarto GE, Sharma S, Stefanick ML, Van Horn L, Wallace RB, Whitlock E, Bassford T, Beresford SA, Black HR, Bonds DE, Brzyski RG, Caan B, Chlebowski RT, Cochrane B, Garland C, Gass M, Hays J, Heiss G, Hendrix SL, Howard BV, Hsia J, Hubbell FA, Jackson RD, Johnson KC, Judd H, Kooperberg CL, Kuller LH, LaCroix AZ, Lane DS, Langer RD, Lasser NL, Lewis CE, Limacher MC, Manson JE and Women's Health Initiative Investigators: Calcium plus vitamin D supplementation and the risk of colorectal cancer. N Engl J Med 354: 684-696, 2006.

16 Keum N, Aune D, Greenwood DC, Ju W and Giovannucci EL: Calcium intake and colorectal cancer risk: dose-response metaanalysis of prospective observational studies. Int J Cancer 135: 1940-1948, 2014.

Received April 3, 2017

Revised April 12, 2017 Accepted April 14, 2017 